\title{
Building additional complexity to in vitro-derived intestinal tissues
}

Samantha A Brugmann $n^{1,2}$ and James M Wells $s^{* 1,3}$

\begin{abstract}
Gastrointestinal (GI) disorders affect up to 25\% of the US population. Common intestinal disorders include malabsorption, irritable bowel syndrome and fecal incontinence. Some Gl disorders such as Hirschsprung's disease have a genetic basis and are associated with an absence or paucity of enteric nerves. Current treatment plans for Gl disorders range from changes in diet to bowel resection, and there are very few drugs available that target the primary deficiencies in intestinal function such as controlled peristalsis. While animal models can recapitulate the broad range of intestinal pathologies of the GI tract, they are intrinsically complicated and of low throughput. Several in vitro systems have been established, and these range from epithelial enteroids to more complex organoids, which contain most intestinal cell types. One of the more complex organoid systems was derived from adult mouse intestines and contains functional enteric nerves and smooth muscle capable of peristalsis. Establishing an equivalent human intestinal system is challenging due to limited access and variable quality of human intestinal tissues. However, owing to recent advances, it is possible to differentiate human induced and embryonic pluripotent stem cells, collectively called pluripotent stem cells, into human intestinal organoids (HIOs) in vitro. Although HIOs contain a significant degree of epithelial and mesenchymal complexity, they lack enteric nerves and thus are unable to model the peristaltic movements of the gut. The goal of this review is to discuss approaches to generate complex in vitro systems that can be used to more comprehensively model common intestinal pathologies. New and more biologically complete human models of the intestine would allow for unprecedented studies of the cellular and molecular basis of normal and pathological gut function. Furthermore, fully functional HIOs could serve as a platform for preclinical drug studies to model absorption and efficacy.
\end{abstract}

Keywords: human induced pluripotent stem cells, embryonic stem cells, neural crest intestine, organoids

\section{Background}

Tissue and functional complexity of the intestine

The small and large intestines have a staggering number of physiological functions, including but not limited to digestion, absorption, endocrine hormone-mediated release of digestive enzymes, peristalsis, satiety and insulin secretion, antigen presentation, control of microbial growth and excretion. To support these physiological functions, the small and large intestines have a diverse set of cell types and a unique tissue architecture (Figure 1a). The intestine is comprised of an inner lumen that is entirely enclosed by a complex epithelium folded into villi aimed at increasing the absorptive surface area. Next to the epithelium is a muscularis mucosa and submucosa

*Correspondence: James.Wells@cchmc.org

'Division of Developmental Biology, Department of Pediatrics, Cincinnati Children's Hospital, 3333 Burnet Avenue, Cincinnati, OH 45229-3026, USA

Full list of author information is available at the end of the article containing a layer of enteric nerves termed the submucosal plexus, which runs the entire length of the small and large intestines. The outer layer of enteric nerves, the myenteric plexus, lies between a layer of circular and longitudinal smooth muscle. In addition, blood vessels are found throughout the layers of the intestine and each villus contains a capillary bed that is involved in uptake of absorbed nutrients and drugs.

There is significant cellular diversity in each layer of the gut. The epithelium is composed of four differentiated cell types (Figure 1b). Most epithelial cells are absorptive enterocytes that transport macromolecules, ions and water. The three remaining cell types are secretory in nature: goblet cells secrete mucins into the lumen that form a protective barrier; Paneth cells secrete antimicrobial peptides such as lysozyme; and enteroendocrine cells secrete hormones that regulate insulin secretion, satiety, motility, and release of digestive enzymes from the gall bladder and pancreas, among other things. The epithelium is highly regenerative and 


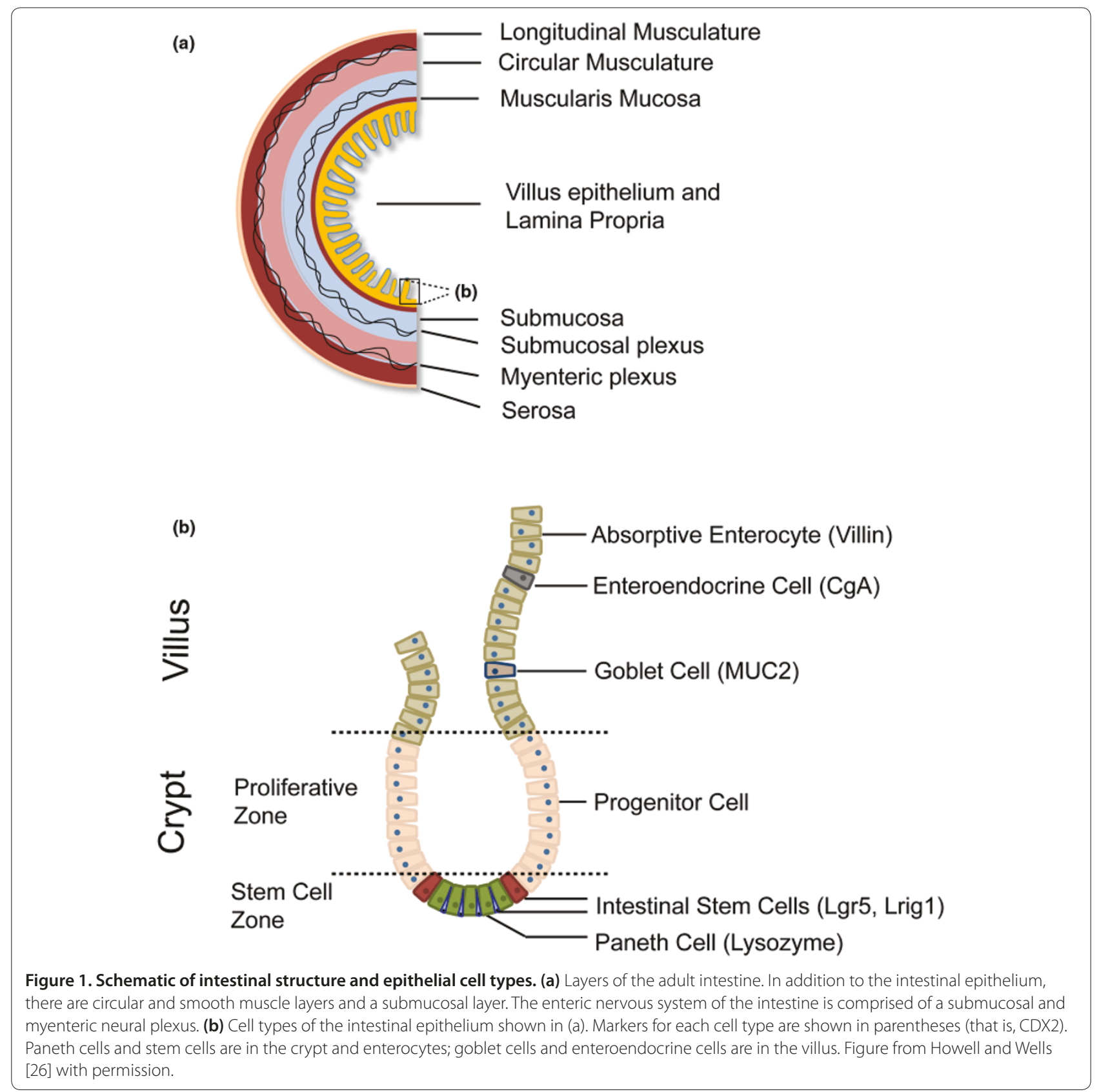

turns over approximately every 7 days. The renewal of the epithelium is driven by intestinal stem cells that reside in the crypts of Lieberkühn at the base of the villi. The submucosal layers contain smooth muscle myocytes, fibroblasts, subepithelial myofibroblasts, as well as enteric ganglia. There is a rudimentary understanding of how the complex architecture and cellular diversity of the intestine arise during embryonic development, which has helped efforts towards directing differentiation of human induced and embryonic pluripotent stem cells, collectively called pluripotent stem cells (PSCs), into the intestine. However, there is still much to be learned.

\section{Intestinal development}

Development of the intestine can be broadly subdivided into several steps including endoderm formation, midgut and hindgut specification, gut tube morphogenesis, assembly of mesenchyme, colonization by neural crest cells, crypt-villus morphogenesis and cytodifferentiation [1] (Figure 2a). Several important signaling pathways have been identified as being required for directing these early stages of intestinal development in a broad range of vertebrate species, including birds, frogs and mice [2-4]. For example, Wnt and fibroblast growth factor signaling pathways direct endoderm into a midgut and hindgut 


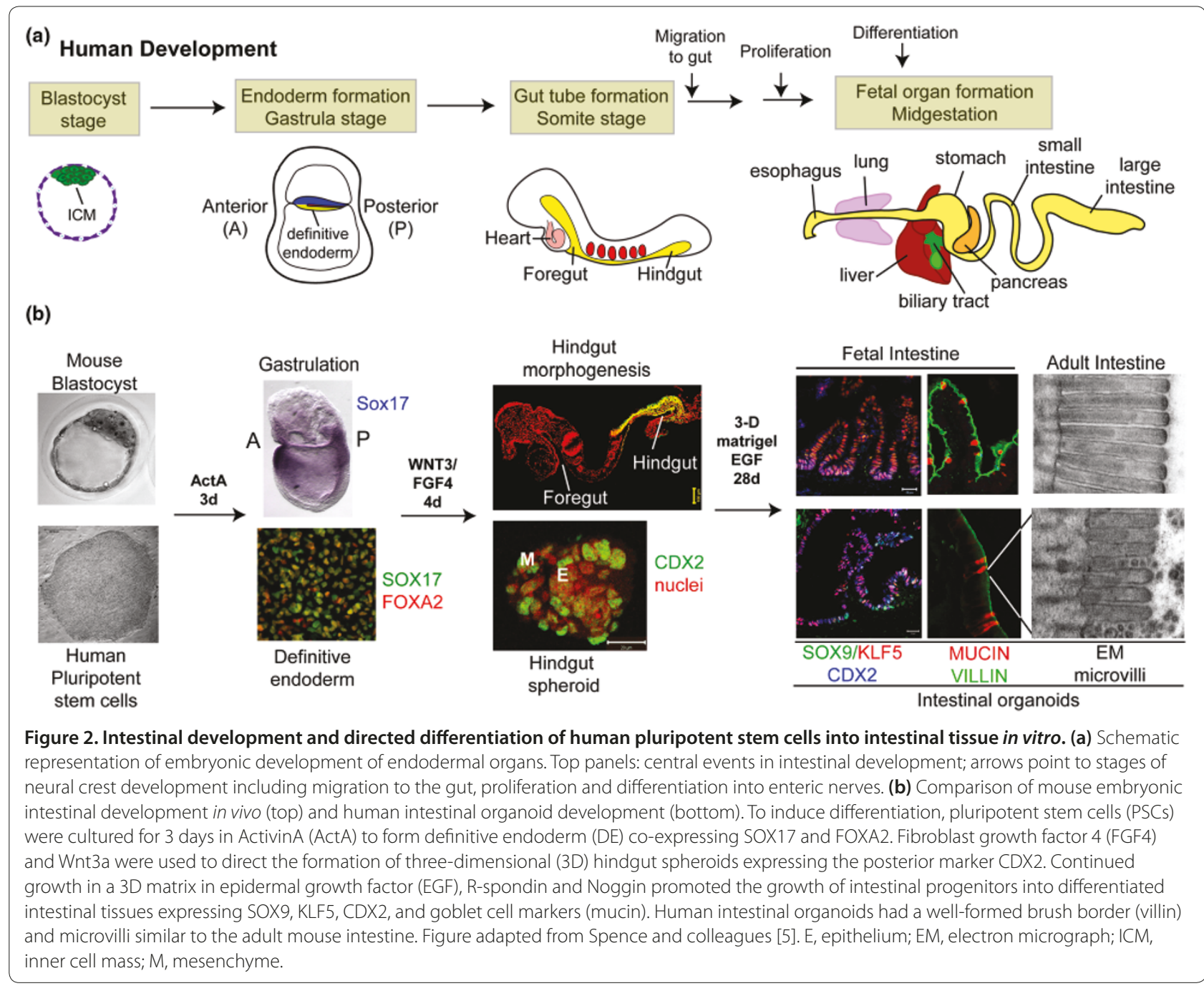

fate, and this process is required for subsequent intestinal development. Moreover, the synergistic activity of both pathways is involved in the initiation of gut tube morphogenesis and formation of the gut mesenchyme [5]. Inhibition of either of these pathways results in abnormal intestinal morphogenesis and the loss of expression of posterior/hindgut markers, including $C d x$ genes. Once formed, the simple cuboidal epithelium of the hindgut and the surrounding mesenchyme undergo a series of reciprocal signaling events resulting in formation of a polarized columnar epithelium containing villi, a proliferative progenitor zone, and distinct intestinal lineages $[5,6]$. Along with development of the epithelium, there is a coordinated development of gut mesenchyme into the submucosal and smooth muscle layers. As discussed below, vagal neural crest cells that give rise to the enteric nervous system (ENS) migrate ventrally, undergo extensive proliferation, and incorporate into the developing gut shortly after gut tube formation $[7,8]$ (Figure 2a).

\section{Future needs and research directions}

Building a better system: engineering additional complexity into human intestinal organoids

Low-throughput, limited genetic diversity, and species differences are recognized limitations for the use of animal models to study gastrointestinal (GI) disease. Recent advances in the understanding of human intestinal stem cells now allows for the derivation of human intestinal epithelial enteroids from patient biopsies [9]. Human enteroids have been cultured from both the small and large intestines and contain all major epithelial cell types. However, enteroids do not contain any additional cell and tissue types such as smooth muscle, supporting fibroblasts, endothelial cells, or enteric nerves. While there are more complex organoid systems derived from adult mouse intestines [10], it would be advantageous to have a more functional human intestinal organoid system as a screening platform to monitor drug absorption and the impact on intestinal motility. 
Generation of more biologically complex human intestinal tissue has been accomplished through the directed differentiation of human PSCs (Figure 2b) $[5,11]$. The process is initiated using the Nodal-related protein activin, which directs differentiation of human PSCs into definitive endoderm [12,13]. Synergistic activity of the fibroblast growth factor and Wnt signaling pathways was then used to promote a posterior gut tube fate [2-4], to induce gut tube morphogenesis, and to promote growth of the intestinal mesenchyme. The resulting gut tube spheroids were strikingly similar to the gut tube of an embryonic day 9 mouse embryo, consisting of a CDX2expressing cuboidal epithelium surrounded by a CDX2expressing mesenchyme. Growth of gut tube spheroids in three-dimensional conditions that favor intestinal growth [14] resulted in the efficient production of human intestinal organoids (HIOs) that have both secretory and absorptive function. Moreover, the cellular diversity and architecture was strikingly similar to that of the developing gut. The epithelium contained crypt and villus-like structures as well as all of the cell types normally found in the gut. The mesenchyme underwent differentiation into stratified layers. Some layers expressed smooth muscle markers whereas others expressed markers of fibroblasts and subepithelial myofibroblasts, both found in the submucosal layer. Furthermore, the HIOs generated via these methods were capable of basic intestinal function, including absorption of amino acids and secretion of mucus, and were able to be passaged in vitro for over 1 year, resulting in 50,000-fold expansion.

Despite the significant level of complexity, PSC-derived HIOs lack both an enteric nervous system and a vascular system. This is probably due to the absence of vascular and ENS precursors, specifically neural crest stem cells. PSCs can be directed to differentiate into vascular and neural crest stem cells [15-19], raising the intriguing possibility that additional tissue complexity can be engineered into HIOs by addition of neural and/or vascular precursors in a contrived manner to recapitulate the normal development of the intestinal vasculature and ENS.

\section{Knowledge gaps}

The ENS is a division of the autonomic nervous system and consists of a nerve plexus that innervates the GI system allowing for peristaltic contractions. Several GI disorders, such as Hirschsprung's disease, are due to an absence or paucity of enteric nerves [20,21]. The ENS is derived from a specialized cell population called the neural crest. The neural crest is a multipotent population of cells that derive from the dorsal neural tube and give rise to a myriad of cell types depending on their anteriorposterior position in the embryo [22]. Anterior/cranial neural crest cells give rise to neurons, bone, and cartilage of the head, whereas posterior/trunk neural crest cells give rise to components of the peripheral nervous system including the ENS. Embryonic studies of cranial neural crest stem cell (NCSC) development have paved the way for recent protocols to direct PSC differentiation into anterior/cranial NCSCs $[15,23]$. However, there are no published methods to direct the differentiation of PSCs into posterior/trunk NCSCs. This lack may be due to the fact that trunk neural crest development, in particular formation of the ENS, is less understood than development of cranial NCSC-derived structures.

There are several reported methods to generate anterior/cranial NCSCs. In one case, ectoderm and neural ectoderm are derived from human ESCs by cellular aggregation or growth in media devoid of signaling molecules that might favor endoderm and mesoderm formation. These conditions result in the formation of neural progenitor cells capable of being further directed into various neural derivatives. For example, exposing neural progenitor cells to factors, such as bone morphogenetic protein, that promote dorsal neural tube fate promotes the formation of cells that have NCSC properties and express NCSC markers, such as p75 [23]. In several reports, human ESC-derived NCSCs were shown to be multipotent and competent to differentiate into an array of NCSC-derived tissues in vitro and in vivo $[15,23,24]$. A similar approach should be possible to generate more posterior/trunk NCSCs, possibly by manipulating anterior-posterior patterning pathways such as retinoic acid or Wnt [25]. Subsequently, it should be possible to integrate human trunk NCSCs into developing intestinal organoids, at a time that approximates normal ENS formation, in attempts to generate HIOs with enteric nerves.

Given our current knowledge of the HIO system, we have identified two major avenues for ongoing research. First, we will need to generate HIOs with more tissue/ biological complexity and functionality. For example, it should be possible to integrate enteric nerves and a capillary plexus into the $\mathrm{HIO}$ system. A more functional HIO would be able to transport luminal factors, such as drugs, across the epithelium and deliver them to an integrated capillary network. The impact of drugs on the ENS and peristalsis could also be evaluated. One approach to building a more complex HIO might be to incorporate vascular precursors and neural crest cells into developing HIOs. Second, as more systems-based approaches are developed, it would be advantageous to integrate HIOs into a microfluidics platform that would allow for precise administration of drugs and other compounds, as well as monitoring of drug absorption and drug impact on intestinal function. Moreover, with an integrated network of microorgan systems on a device, 
it should be possible to measure the impact of intestinal function on drug bioavailability and the effects on other organ systems. The ability to perform this type of testing on a high-throughput scale, with PSC lines from a broad genetic background, could significantly improve our predictions of drug toxicity and efficacy in clinical trials.

\section{Abbreviations}

ENS, enteric nervous system; GI, gastrointestinal; $\mathrm{HIO}$, human intestinal organoid; NCSC, neural crest stem cell; PSC, pluripotent stem cell.

\section{Competing interests}

The authors declare that they have financial interests in Patent application 0088544-008PRO.

\section{Acknowledgments}

The authors acknowledge National Institutes of Health grants R01DK092456, R01DK080823 and U18 TR000546 for funding related to the research described in this review. The publication costs for this article were funded by National Institutes of Health grant U18 TR000546.

\section{Declarations}

Publication of this supplement has not been supported by sponsorship. Articles have undergone the journal's standard review process. The Editors declare that they have no competing interests.

This article has been published as part of Stem Cell Research \& Therapy Volume 4 Supplement 1, 2013: Stem cells on bioengineered microphysiological platforms for disease modeling and drug testing. The full contents of the supplement are available online at http://www.stemcellres.com/supplements/4/S1.

\section{Author details}

'Division of Developmental Biology, Department of Pediatrics, Cincinnati Children's Hospital, 3333 Burnet Avenue, Cincinnati, OH 45229-3026, USA ${ }^{2}$ Division of Plastic Surgery, Department of Surgery, Cincinnati Children's Hospital, 3333 Burnet Avenue, Cincinnati, OH 45229-3026, USA. ${ }^{3}$ Division of Endocrinology, Department of Pediatrics, Cincinnati Children's Hospital, 333 Burnet Avenue, Cincinnati, OH 45229-3026, USA.

Published: 20 December 2013

\section{References}

1. Spence JR, Lauf R, Shroyer NF: Vertebrate intestinal endoderm development. Dev Dyn 2011, 240:501-520.

2. Dessimoz J, Opoka R, Kordich JJ, Grapin-Botton A, Wells JM: FGF signaling is necessary for establishing gut tube domains along the anterior-posterior axis in vivo. Mech Dev 2006, 123:42-55.

3. McLin VA, Rankin SA, Zorn AM: Repression of Wnt/ $\beta$-catenin signaling in the anterior endoderm is essential for liver and pancreas development. Development 2007, 134:2207-2217.

4. Wells JM, Melton DA: Early mouse endoderm is patterned by soluble factors from adjacent germ layers. Development 2000, 127:1563-1572.

5. Spence JR, Mayhew CN, Rankin SA, Kuhar MF, Vallance JE, Tolle K, Hoskins EE, Kalinichenko WV, Wells SI, Zorn AM, Shroyer NF, Wells JM: Directed differentiation of human pluripotent stem cells into intestinal tissue in vitro. Nature 2011, 470:105-109.

6. de Santa Barbara P, van den Brink GR, Roberts DJ: Development and differentiation of the intestinal epithelium. Cell Mol Life Sci 2003, 60:1322-1332

7. Epstein ML, Poulsen KT, Thiboldeaux R: Formation of ganglia in the gut of the chick embryo. J Comp Neurol 1991, 307:189-199.

8. Gershon MD, Chalazonitis A, Rothman TP: From neural crest to bowel: development of the enteric nervous system. J Neurobio/ 1993, 24:199-214.
9. Sato T, Stange DE, Ferrante M, Vries RG, Van Es JH, Van den Brink S, Van Houdt WJ, Pronk A, Van Gorp J, Siersema PD, Clevers H: Long-term expansion of epithelial organoids from human colon, adenoma, adenocarcinoma, and Barrett's epithelium. Gastroenterology 2011, 141:1762-1772.

10. Ootani A, Li X, Sangiorgi E, Ho QT, Ueno H, Toda S, Sugihara H, Fujimoto K, Weissman IL, Capecchi MR, Kuo CJ: Sustained in vitro intestinal epithelial culture within a Wnt-dependent stem cell niche. Nat Med 2009, 15:701-706.

11. MCCracken KW, Howell JC, Wells JM, Spence JR: Generating human intestinal tissue from pluripotent stem cells in vitro. Nat Protoc 2011, 6:1920-1928.

12. D'Amour KA, Agulnick AD, Eliazer S, Kelly OG, Kroon E, Baetge EE: Efficient differentiation of human embryonic stem cells to definitive endoderm. Nat Biotechnol 2005, 23:1534-1541

13. Spence JR, Wells JM: Translational embryology: using embryonic principles to generate pancreatic endocrine cells from embryonic stem cells. Dev Dyn 2007, 236:3218-3227

14. Sato T, Vries RG, Snippert HJ, van de Wetering M, Barker N, Stange DE, van Es $\mathrm{JH}$, Abo A, Kujala P, Peters PJ, Clevers H: Single Lgr5 stem cells build cryptvillus structures in vitro without a mesenchymal niche. Nature 2009, 459:262-265.

15. Bajpai R, Chen DA, Rada-Iglesias A, Zhang J, Xiong Y, Helms J, Chang CP, Zhao Y, Swigut T, Wysocka J: CHD7 cooperates with PBAF to control multipotent neural crest formation. Nature 2010, 463:958-962.

16. Lee $G$, Chambers SM, Tomishima MJ, Studer L: Derivation of neural crest cells from human pluripotent stem cells. Nat Protoc 2010, 5:688-701.

17. Levenberg S, Golub JS, Amit M, Itskovitz-Eldor J, Langer R: Endothelial cells derived from human embryonic stem cells. Proc Natl Acad Sci U S A 2002, 99:4391-4396.

18. Liu Q, Spusta SC, Mi R, Lassiter RN, Stark MR, Hoke A, Rao MS, Zeng X: Human neural crest stem cells derived from human ESCs and induced pluripotent stem cells: induction, maintenance, and differentiation into functional schwann cells. Stem Cells Trans/ Med 2012, 1:266-278.

19. Menendez L, Yatskievych TA, Antin PB, Dalton S: Wnt signaling and a Smad pathway blockade direct the differentiation of human pluripotent stem cells to multipotent neural crest cells. Proc Natl Acad Sci U S A 2011, 108:19240-19245.

20. Chan KK, Wong CK, Lui VC, Tam PK, Sham MH: Analysis of SOX10 mutations identified in Waardenburg-Hirschsprung patients: differential effects on target gene regulation. J Cell Biochem 2003, 90:573-585.

21. Pingault V, Bondurand N, Kuhlbrodt K, Goerich DE, Prehu M-O, Puliti A, Herbarth B, Hermans-Borgmeyer I, Legius E, Matthijs G, Amiel J, Lyonnet S, Ceccherini I, Romeo G, Smith JC, Read AP, Wegner M,Goossens M: Sox10 mutations in patients with Waardenburg-Hirschsprung disease. Nat Genet 1998, 18:171-173.

22. Bronner ME, LeDouarin NM: Development and evolution of the neural crest: an overview. Dev Biol 2012, 366:2-9.

23. Lee G, Kim H, Elkabetz Y, Al Shamy G, Panagiotakos G, Barberi T, Tabar V, Studer $L$ : Isolation and directed differentiation of neural crest stem cells derived from human embryonic stem cells. Nat Biotechnol 2007, 25:1468-1475.

24. Clewes O, Narytnyk A, Gillinder KR, Loughney AD, Murdoch AP, Sieber-Blum M: Human epidermal neural crest stem cells (hEPI-NCSC) characterization and directed differentiation into osteocytes and melanocytes. Stem Cell Rev 2011, 7:799-814.

25. Kudoh T, Wilson SW, Dawid IB: Distinct roles for Fgf, Wnt and retinoic acid in posteriorizing the neural ectoderm. Development 2002, 129:4335-4346.

26. Howell JC, Wells JM: Generating intestinal tissue from stem cells: potential for research and therapy. Regen Med 2011, 6:743-755.

\section{doi:10.1186/scrt362}

Cite this article as: Brugmann SA, Wells JM: Building additional complexity to in vitro-derived intestinal tissues. Stem Cell Research \& Therapy 2013, 4(Suppl 1):S1. 